\title{
Distances to five resolved galaxies in the Canes Venatici cloud
}

\author{
I.D. Karachentsev ${ }^{1}$ and I.O. Drozdovsky ${ }^{2}$ \\ 1 Special Astrophysical Observatory, Russian Academy of Sciences, N. Arkhyz, Stavropolsky kraj, KChR, 357147, Russia \\ 2 Astronomical Institute, St.-Petersburg State University, Petrodvoretz, 198904, Russia
}

Received September 9, 1997; accepted January 5, 1998

\begin{abstract}
We present results of CCD imaging in $B, V$ of five late-type galaxies with radial velocities $V_{0}<350 \mathrm{~km} / \mathrm{s}$ in the Canes Venatici cloud. Based on the photometry of their brightest blue stars we derived the following distances to the galaxies: $9.7 \mathrm{Mpc}$ for NGC 4144, 4.5 Mpc for NGC 4244, 4.2 Mpc for NGC 4395, 2.9 Mpc for NGC 4449, and $8.2 \mathrm{Mpc}$ for UGC $8331^{1}$.
\end{abstract}

Key words: galaxies: distances - galaxies: NGC 4144; NGC 4244; NGC 4395; NGC 4449; UGC 8331

\section{Introduction}

The nearest scattered cloud in Canes Venatici contains about fifty spiral and irregular galaxies with corrected radial velocities $V_{0}<500 \mathrm{~km} / \mathrm{s}$. Before 1996 only four galaxies of this complex: IC 4182, DDO 154, DDO 168, and UGC 8508 had direct distance estimates via photometry of their brightest stars (Sandage \& Tammann 1982; Carignan \& Beaulieu 1989; Bresolin et al. 1993; Karachentsev et al. 1994). Georgiev et al. (1997), Makarova et al. (1997), Makarova et al. (1998), and Tikhonov \& Karachentsev (1998) increased this number up to 38 objects. Among other unstudied members of the CVn cloud some galaxies belong to early types E-Sa, for which the brightest stars method cannot be applied for distance determination. The remaining galaxies of the complex are well resolved into stars. Here we consider five rather large galaxies: NGC 4144, NGC 4244, NGC 4395, NGC 4449, and UGC 8331, whose distances are not found in the literature. The first three of them are represented in the "Atlas of Galaxies Useful for Measuring the Cosmologic Distance Scale" (Sandage \& Bedke 1988).

\footnotetext{
1 Tables 3 to 7 are only available in electronic form at the CDS via anonymous ftp to cdsarc.u-strasbg.fr (130.79.128.5) or via http://cdsweb.u-strasbg.fr/Abstract.html
}

Table 1. Observational log

\begin{tabular}{|cccc|}
\hline Object & Date & Filter & Exp.(s) \\
\hline NGC 4144 & Feb. 4 & $B$ & 600 \\
& & $V$ & 600 \\
& & $I$ & 600 \\
NGC 4244n & Feb. 6 & $B$ & 600 \\
& & $V$ & 300 \\
NGC 4244s & Feb. 6 & $B$ & 600 \\
& & $V$ & 300 \\
NGC 4395 & Feb. 4 & $B$ & 600 \\
& & $V$ & 600 \\
NGC 4449 & \multirow{2}{*}{ Feb. 4} & $B$ & 300 \\
& & $V$ & 300 \\
& & $I$ & 300 \\
UGC 8331 & Feb. 6 & $B$ & 600 \\
& & $V$ & 300 \\
\hline
\end{tabular}

\section{Observations and photometry}

The galaxies were observed on February 4-6, 1995 with a CCD camera of the 2.56 meter Nordic telescope at La Palma with a seeing of $0.7-1.3$ arcsec. A TK CCD chip provided a view field of $3 \times 3^{\prime}$ with a resolution of $0.176^{\prime \prime} /$ pixel. The exposure times in different filters are shown in Table 1 for each galaxy. Equatorial standard stars from Landolt (1992) were observed for calibration. Photometry of stellar objects in the galaxies were carried out with MIDAS using ALLSTAR DAOPHOT package (Stetson 1987). The frames were debiased, darksubtracted and flat-fielded. After transfering instrumental magnitudes into the standard Johnson-Cousins system we derived $B, V, I$ magnitudes with a typical error which is expected to be about $0.05 \mathrm{mag}$. In the case of the galaxy NGC 4449, having a very crowded stellar field, we repeated photometry after subtracting its frame smoothed with a median filter. The results of our stellar photometry are presented in Tables $3-7$, which can be found in a computer-readable form at the CDS. 


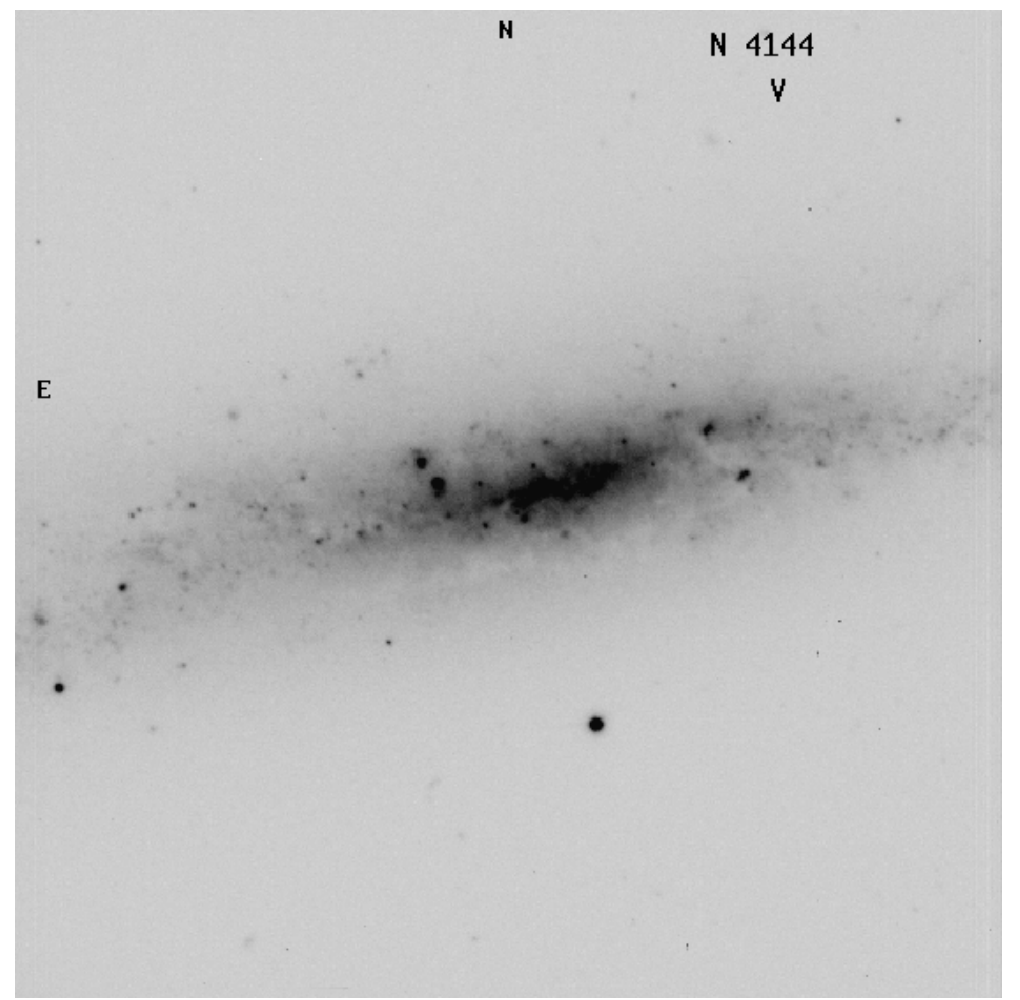

Fig. 1. The $V$ frame of NGC 4144. As for all the other images, North is top, and East is left. The upper right corner corresponds to $X=0, Y=0$ of the frame coordinates given in the Tables 2-6, i.e. $X$ increases to East and $Y$ to South

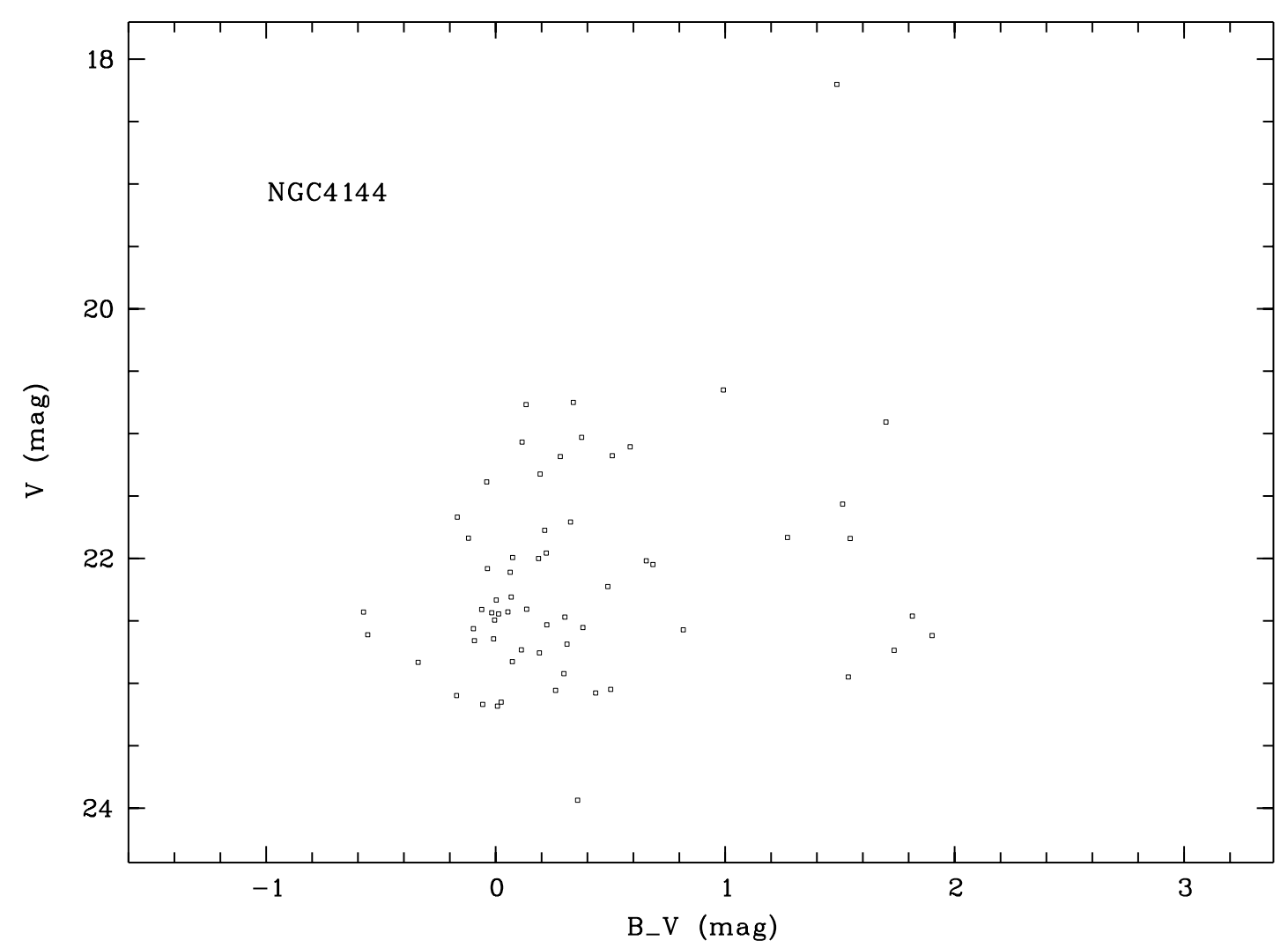

Fig. 2. $V$ vs. $(B-V)$ diagram for NGC 4144 


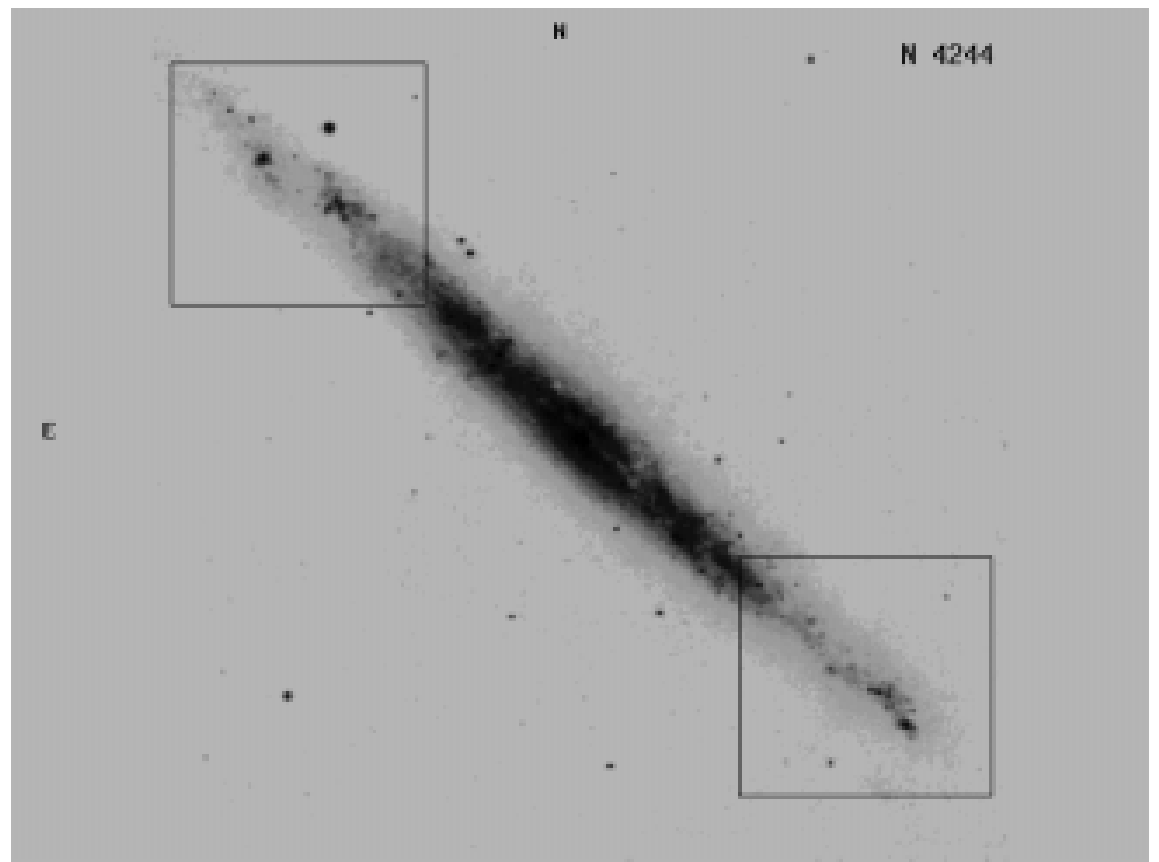

Fig. 3. A view of NGC 4244 from the GCO NGC Archives. Two squares correspond to the Nordic CCD frames

\section{Distance estimates for the galaxies}

\section{1. $N G C 4144$}

According to RC3 (de Vaucouleurs et al. 1991), this Scdtype galaxy has a total magnitude $B_{\mathrm{T}}=12.01$ and angular dimensions $6.1^{\prime} \times 1.5^{\prime}$, extending a bit beyond our CCD frame (Fig. 1). Out of the stellar-like objects selected by DAOPHOT in the frame field we put in Table 3 only 62 stars, whose image parameters satisfy the conditions: $\sigma(V)<0.2 \mathrm{mag},|\mathrm{SHARP}|<2,|\mathrm{CHI}|<2$. The colourmagnitude diagram (=CMD) for them is shown in Fig. 2. Following the tradition (Sandage \& Tammann 1974; de Vaucouleurs 1978), we selected as blue supergiant candidates the stars with colour indexes: $B-V<0.4$, and $V-I<0.5$. The distance modulus to a galaxy was determined via the relation

$\mu(B)=1.51 \cdot<B(3 B)>-0.51 B_{\mathrm{T}}-A_{\mathrm{b}}+4.14$,

where $\langle B(3 B)>$ indicates the average apparent magnitude of three brightest stars, $A_{\mathrm{b}}$ is the galactic extinction in $B$. The numerical coefficients were derived (Karachentsev \& Tikhonov 1994) from galaxies with distances known via cepheids. For the three brightest blue stars (\#42, 25, and 59) we obtained $<B(3 B)>=$ $21.15,\langle B-V\rangle=0.07$, and $\langle V-I\rangle=0.18$, which gives with $A_{\mathrm{b}}=0.02$ a distance modulus of 29.93 mag or $D=9.7 \mathrm{Mpc}$.

On the CMD one can see some red stars with the colours $B-V>1.6$ and $V-I>2.0$, which is typical of red supergiants. However, the brightest of them are situated outside the galaxy boundary, and for fainter ones, with $B>24 \mathrm{mag}$, the colour measurement error is too large to consider them as real red supergiant candidates.

\section{2. $N G C 4244$}

Having angular dimensions $16^{\prime} \times 1.8^{\prime}$, this edge-on galaxy extends far beyond a single CCD frame. This is why we obtained separately two pairs of $B, V$ frames for the northern and southern edges of NGC 4244, where the density of stars looks less crowded allowing more reliable photometry. A picture of the entire galaxy taken from the GCO NGC Archives is shown in Fig. 3, where two squares indicate the position of our CCD frames. The $V$ images of these regions are presented in Fig. 4. Table 4 contains the photometry results for 73 stars in the northern frame as well for 48 stars in the southern one just with the same restrictions on their $\sigma(V),|\mathrm{SHARP}|$, and $|\mathrm{CHI}|$ as the previous case. The CM diagram for these stars is shown in Fig. 5. We note that blue stars predominate amongst the brightest ones in agreement with the presence of bright blue complexes in the galaxy disk. For the three brightest stars within the northern and southern frames we obtained the mean apparent magnitudes: 20.11 and $20.23 \mathrm{mag}$, respectively. Combination of the two subsamples increases the mean to 19.88 , i.e. by -0.29 mag. The central more crowded region of NGC 4244, occupying about the same area as our CCD frames, may contain blue supergiant candidates too. We assume that taking into account the remaining (unobserved) half of the galaxy leads another similar correction, $-0.29 \mathrm{mag}$. Therefore we adopt $\langle B(3 B)\rangle=19.59 \mathrm{mag}$ as the mean magnitude of the three brightest stars for the whole galaxy. With 


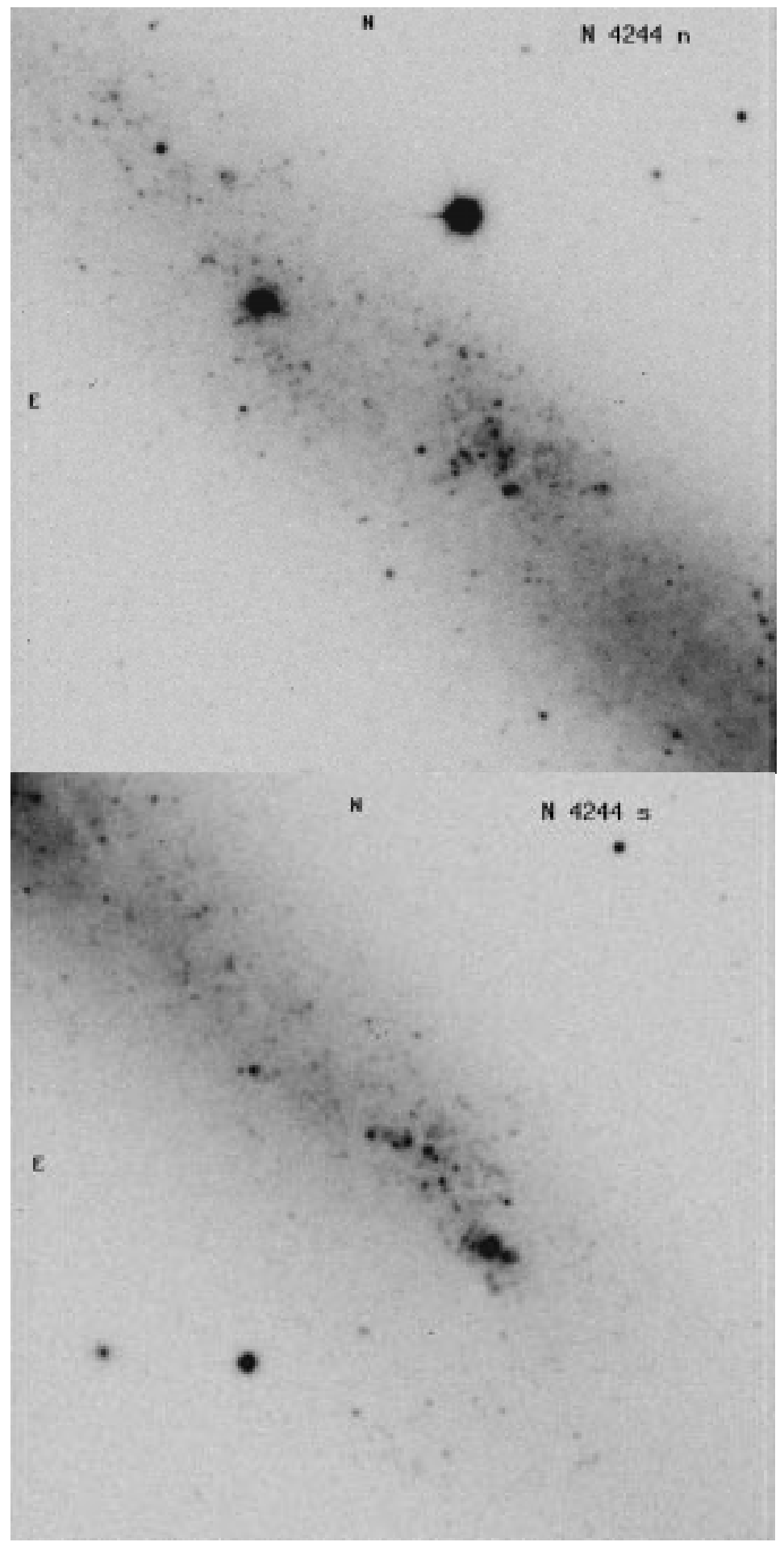

Fig. 4. The $V$ frames of northern a) and southern b) parts of NGC 4244

$B_{\mathrm{T}}=10.67$ it gives for NGC 4244 a distance modulus of $28.28 \mathrm{mag}$ or $D=4.53 \mathrm{Mpc}$. Note that this is somewhat more uncertain than for the previous galaxy. In this galaxy we found no appropriate candidates to be red supergiants.

\section{3. $N G C 4395$}

This Sd-type galaxy has a total apparent magnitude $B_{\mathrm{T}}=10.61$ and angular dimensions $13^{\prime} \times 10^{\prime}$. According to Fillipenko \& Sargent (1989) it is the nearest representator of a galaxy with the Seyfert 1 type nucleus. On the photograph presented in the Atlas by Sandage \& Bedke (1988) it is well resolved into stars. The most 


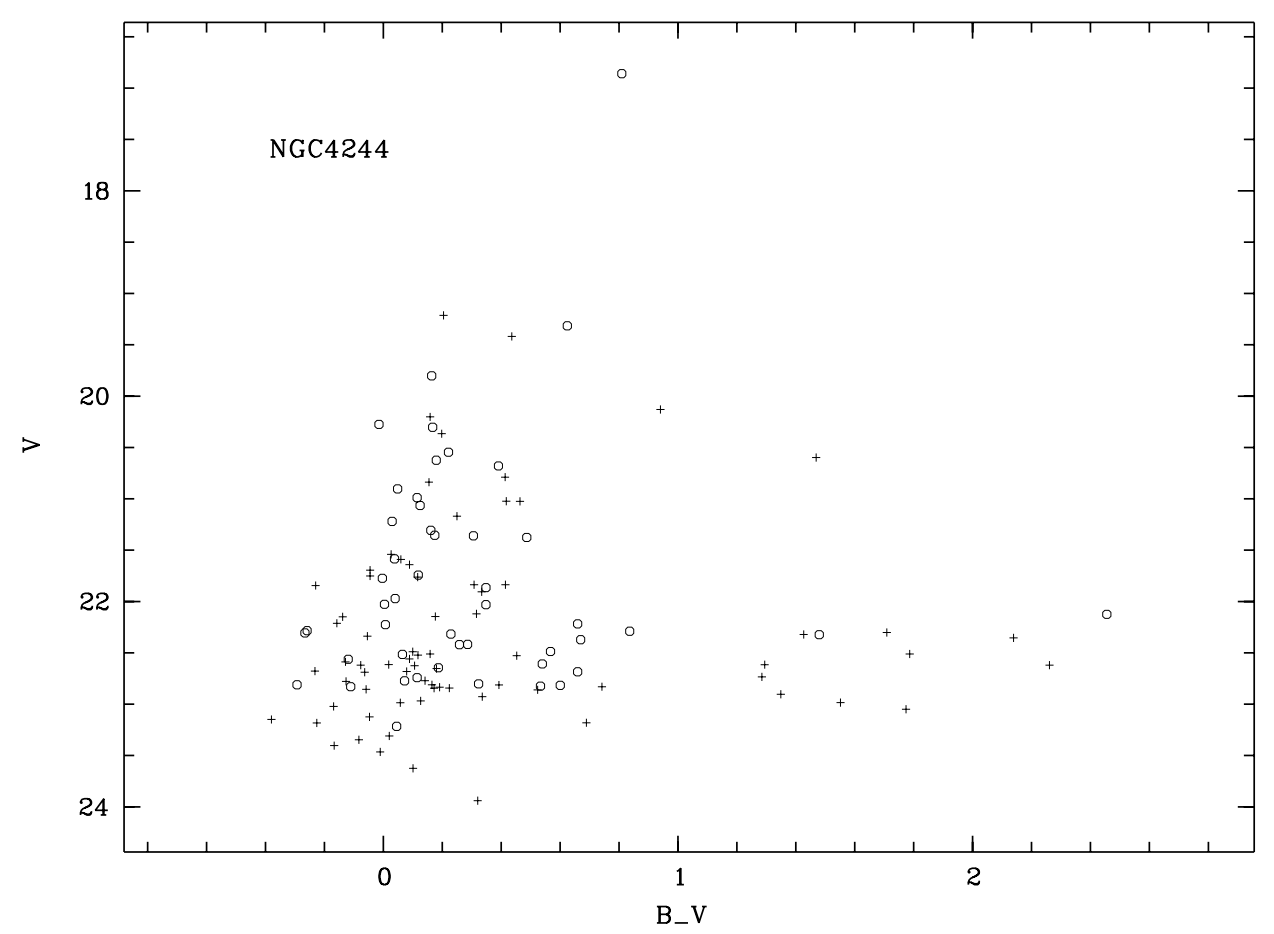

Fig. 5. CM diagram for 73 stars at the northern (crosses) and 48 stars at the southern (circles) edges of NGC 4244

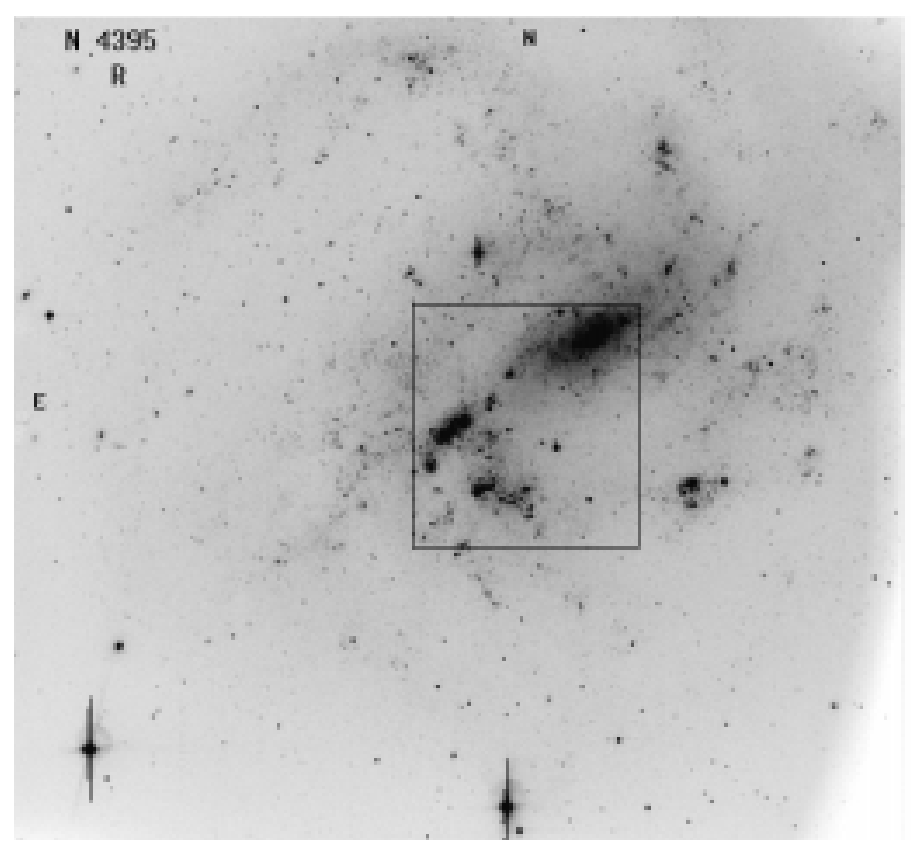

Fig. 6. A view of NGC 4395 in $R$ band. The frame was obtained with the Isaac Newton Telescope CCD camera on 26.04.1992. The square indicates the position of our CCD frame 


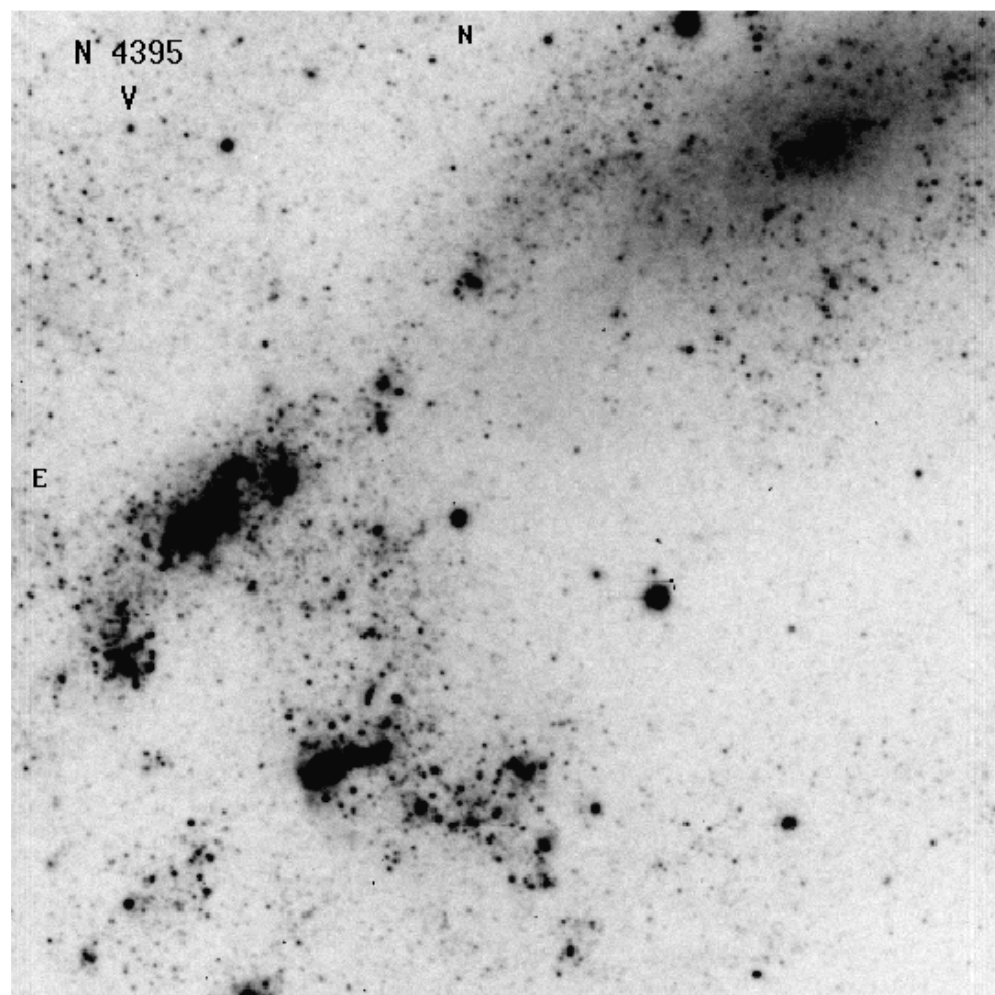

Fig. 7. The $V$ Nordic frame of the SE part of NGC 4395

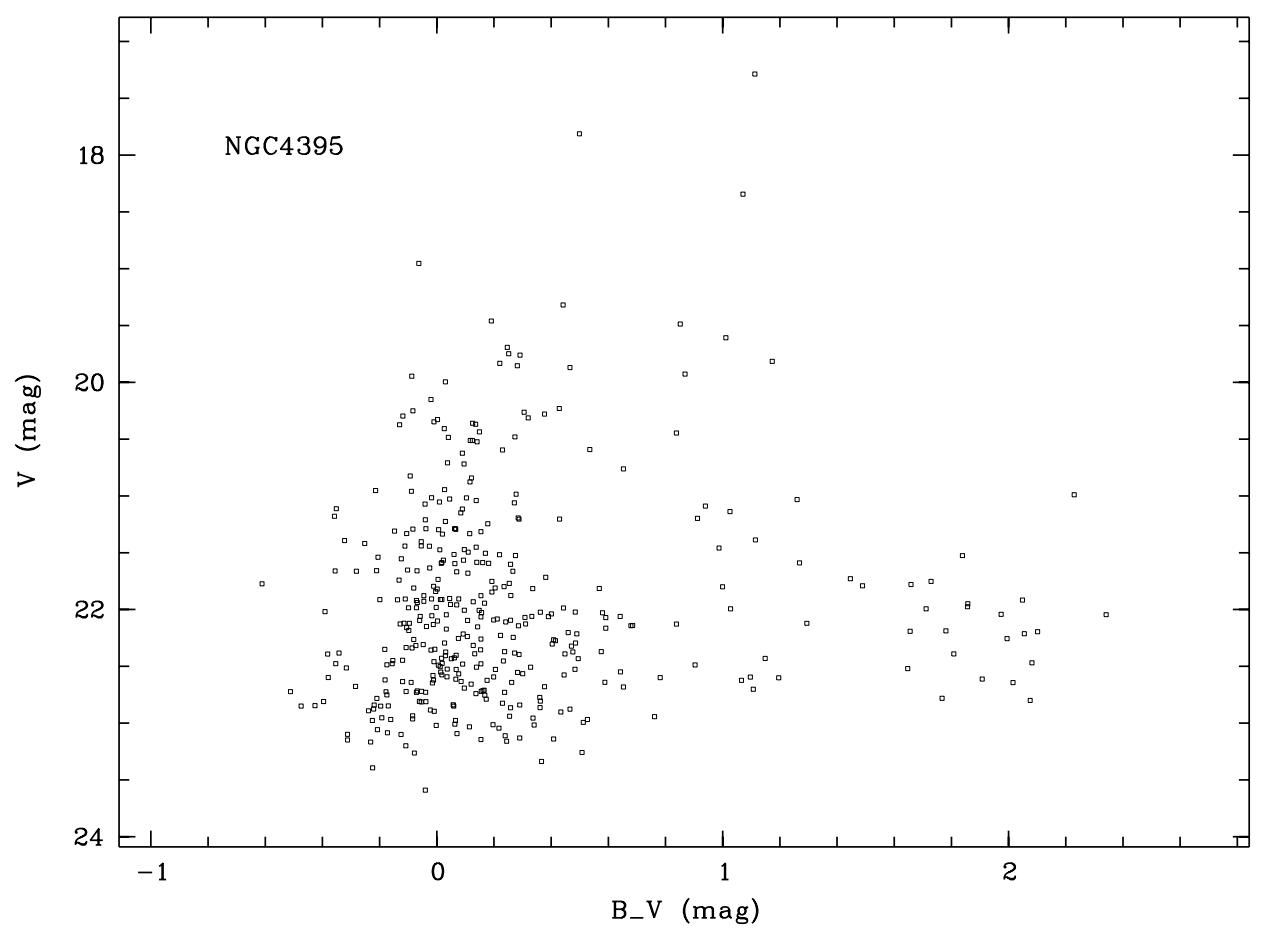

Fig. 8. CM diagram for 389 stars in NGC 4395 


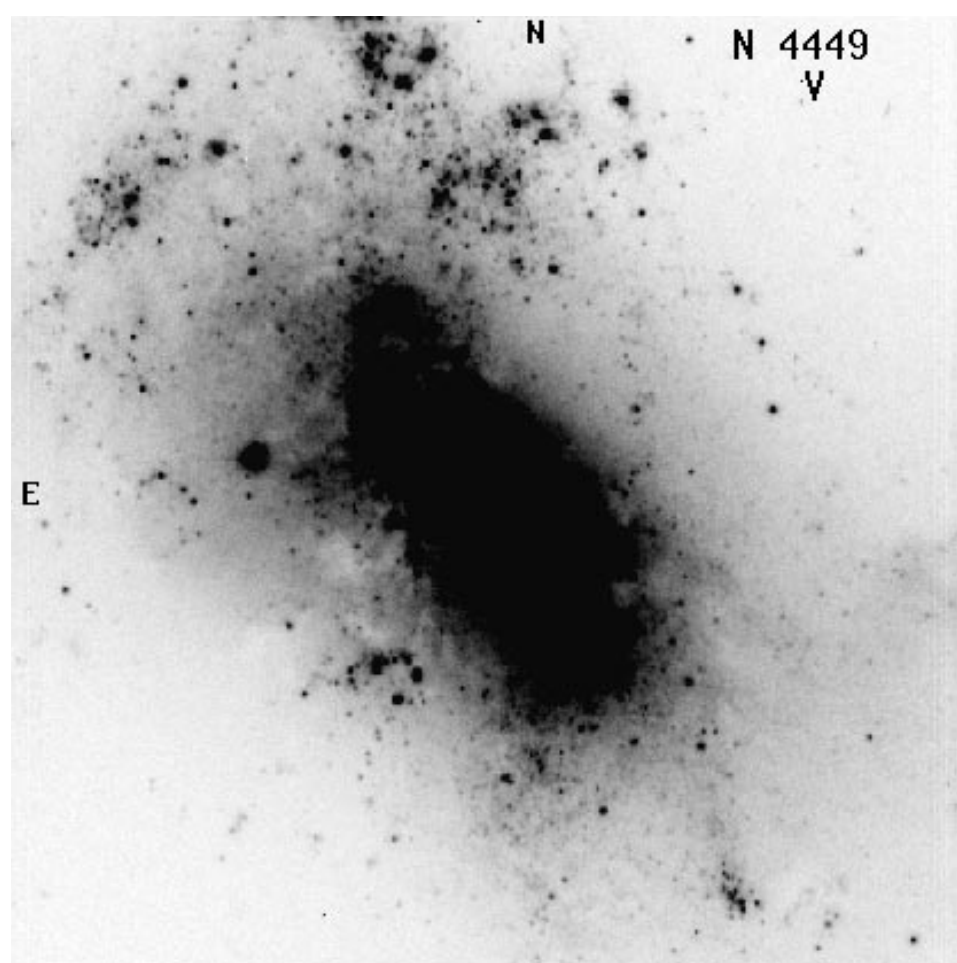

Fig. 9. The $V$ frame of NGC 4449

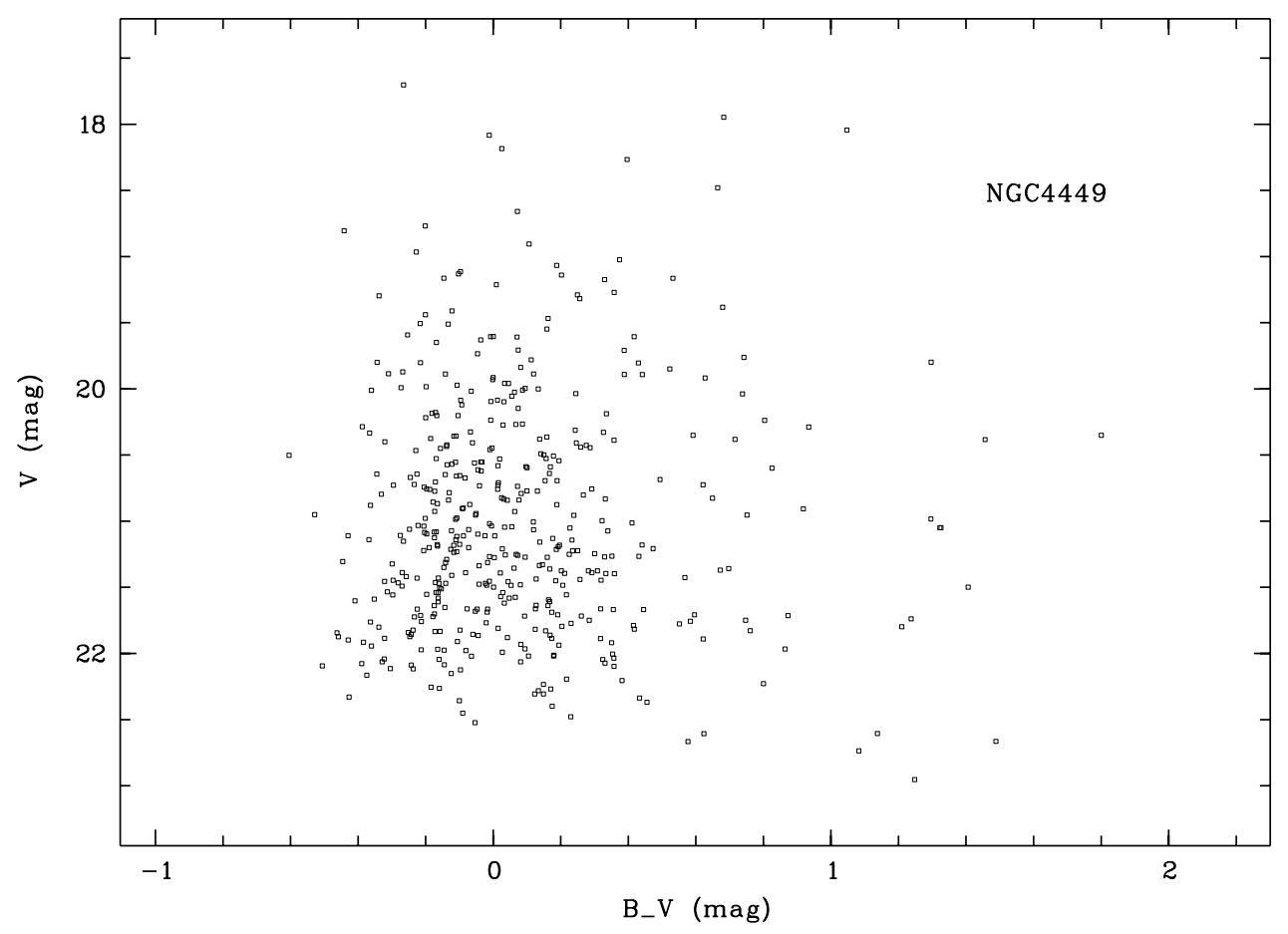

Fig. 10. $V$ vs. $(B-V)$ diagram for 448 stars in NGC 4449 


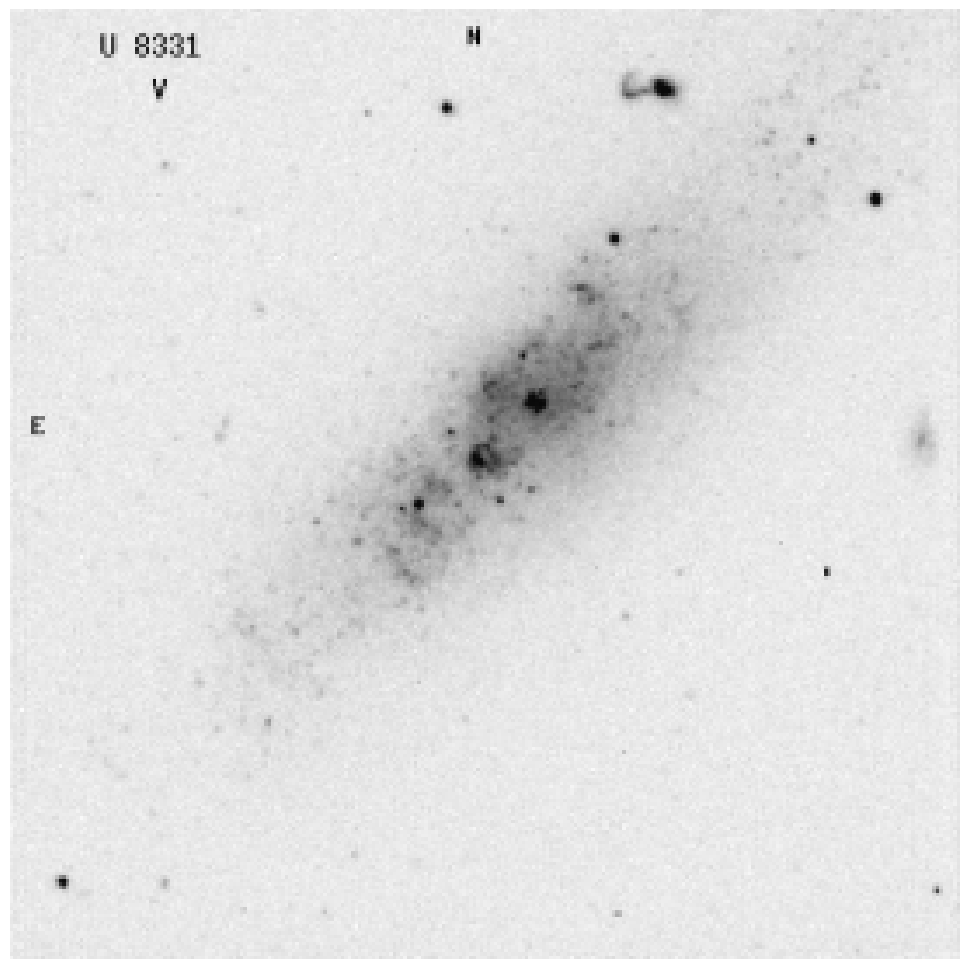

Fig. 11. The $V$ frame of UGC 8331

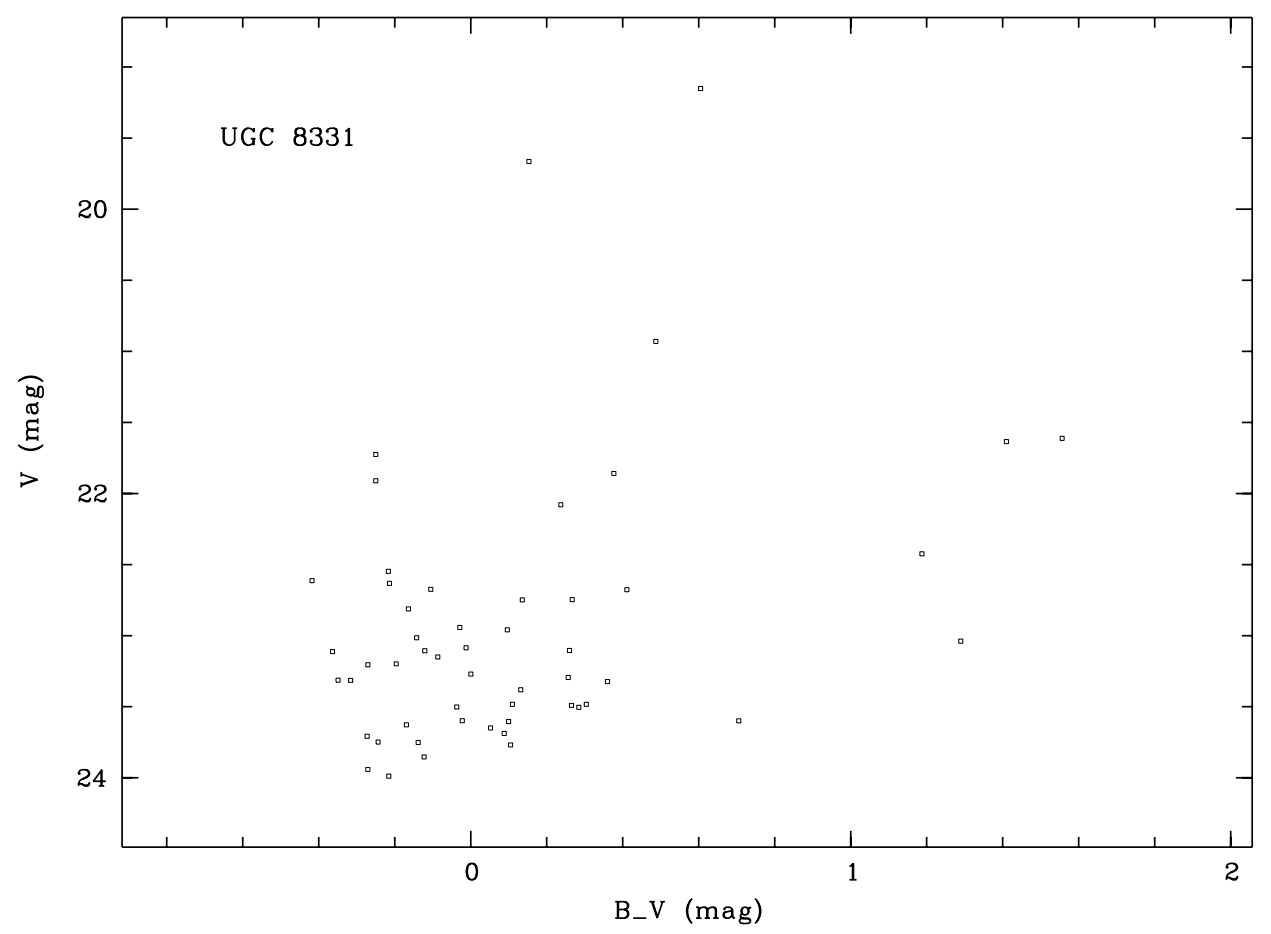

Fig. 12. CM diagram for 53 stars in UGC 8331 
Table 2. Basic parameters of the CVn galaxies

\begin{tabular}{|l|rrrrr|}
\hline Parameter & $\mathrm{N} 4144$ & $\mathrm{~N} 4244$ & $\mathrm{~N} 4395$ & $\mathrm{~N} 4449$ & $\mathrm{U} 8331$ \\
\hline$a_{25} \times b_{25},($ arcmin $)$ & $6.1 \times 1.5$ & $16.0 \times 1.8$ & $13.0 \times 10$ & $6.3 \times 4.9$ & $2.5 \times 0.9$ \\
$B_{\mathrm{T}},(\mathrm{mag})$ & 12.01 & 10.67 & 10.61 & 9.83 & 14.61 \\
$A_{B},(\mathrm{mag})$ & 0.02 & 0.01 & 0.00 & 0.02 & 0.02 \\
$V_{0},(\mathrm{~km} / \mathrm{s})$ & +319 & +255 & +315 & +251 & +346 \\
$\langle B(3 B)>,(\mathrm{mag})$ & 21.15 & {$[19.59]$} & 19.47 & 18.68 & 21.78 \\
$\left\langle B-V>{ }_{3}\right.$ & +0.07 & +0.12 & +0.01 & -0.12 & -0.31 \\
$\mu(3 B),(\mathrm{mag})$ & 29.93 & 28.28 & 28.13 & 27.33 & 29.58 \\
$D,(\mathrm{Mpc})$ & 9.69 & 4.53 & 4.22 & 2.93 & 8.23 \\
$M_{B},(\mathrm{mag})$ & -17.94 & -17.62 & -17.52 & -17.52 & -14.99 \\
\hline
\end{tabular}

prominent stellar complexes are situated on its SE side. This region was chosen by us to search for blue supergiants. Fig. 6 shows the general view of NGC 4395 taken from the Royal Greenwich Observatory, Isaak Newton Group Archives, where our CCD frame boundary is indicated by a square. The $V$ frame of the observed region is reproduced in Fig. 7. The ALLSTAR photometry results for 389 stars are given in Table 5. The CM diagram derived from these data (Fig. 8) confirms the general impression that an active star-formation process is going in the galaxy, producing a lot of bright blue stars. Adopting for the three brightest ones (\# 317, 369, 207) a mean $\langle B(3 B)\rangle=19.47$ yields a distance modulus of 28.13 mag. Probably, there are some other brighter supergiants beyond our CCD frame. Consequently the derived value of distance is suggested to be an upper limit.

\section{4. $N G C 4449$}

This irregular galaxy with $B_{\mathrm{T}}=9.83$ and a standard diameter of 6.3 has a rather high surface brightness and a box-like shape. According to Bajaja et al. (1994), a huge HI envelope around it extends up to 12 times the optical diameter reaching a total diameter of $75^{\prime}(!)$. The central part of NGC 4449 is reproduced in Fig. 9 from our V CCD frame. Due to the strong stellar crowding, the presence of dust clouds, and the high brightness gradients, stellar photometry is difficult in the galaxy. Application of ALLSTAR DAOPHOT to the initial $B, V$ frames yields us magnitudes for 998 stellar-like objects with $|\mathrm{SHARP}|<2$, $|\mathrm{CHI}|<2$, and $\sigma(V)<0.2 \mathrm{mag}$. However, part of them have an ubnormal combination of $B-V$ and $V-I$ colours, which may be related to hidden photometric errors which are caused by the inhomogeneous galaxy background. For this reason we repeated ALLSTAR photometry of the images after subtraction from its of a frame smoothed with a median filter of a 31-pixel round window diameter. As a result we present in Table 6 only 448 stars, whose apparent $V$ magnitudes are within $0.2 \mathrm{mag}$ from the initial ALLSTAR ones. Nevertheless, even this condition does not ensure a high photometric accuracy in this overcrowded stellar field. The CM diagram of the stars from Table 6 is presented in Fig. 10. We suppose that some compact HII regions and multiple stars (for example, \#32 and 340) may occur among the brightest blue objects. Adopting the stars: \#317, 339, and 229 as the brightest supergiant candidates we obtain for them $\langle B(3 B)\rangle=$ $18.68 \mathrm{mag},\langle B-V\rangle=-0.12,\langle V-I\rangle=-0.24$, which yields a distance modulus of $27.33 \mathrm{mag}$ or $D=2.93 \mathrm{Mpc}$.

\section{5. $U G C 8331=D D O 169$}

This irregular dwarf galaxy from the list of van den Bergh (1966) is not as impressive as the objects considered previously. Its $V$ image is shown in Fig. 11. According to our measurements DDO 169 has a total apparent magnitude and colour: $B_{\mathrm{T}}=14.61$ and $(B-V)_{\mathrm{T}}=0.39$. The results of the photometry of 53 stars are presented in Table 7 and on the CM diagram (Fig. 12). Via the three brightest blue stars with $\langle B(3 B)\rangle=21.78$ we obtain a distance modulus of $29.58 \mathrm{mag}$ or $D=8.23 \mathrm{Mpc}$.

Note that north of UGC 8331 there is a compact object of unusual shape with a narrow curved tail. Its total magnitude is $B=19.62$ and $B-V=0.92$. In the central region of the object we distinguish two knots or nuclei separated by 0.7 . Probably, this is a pair of distant merging galaxies.

\section{Summary}

Some basic parameters of the considered galaxies are collected in Table 2. Its lines contain: (1) - the standard major and minor diameters of the galaxy from the RC3; (2) — the total apparent magnitude; (3) — the adopted value of galactic extinction; (4) — the radial velocity with respect to the centroid of the Local Group; $(5,6)$ - the mean magnitude and mean colour of the three brightest blue stars; $(7,8)$ - the photometric distance modulus and corresponding linear distance; (9) - the absolute magnitude of the galaxy.

As one can see from these data the radial velocities of all 5 galaxies lie in the narrow interval: $[+250,+350]$ $\mathrm{km} / \mathrm{s}$. At the same time their photometric distance estimates differ by more than a factor 3. Apparently, this indicates significant peculiar (non-Hubble) motions inside 
the Canes Venatici cloud. We intend to discuss the kinematics of this nearby complex when the program of measurements of individual distances to galaxies in CVn is completed.

Acknowledgements. The authors thank Leo Takalo and Pekka Heinämäki for their participation in the observations. This work is supported by INTAS-RFBR grant N95-IN-RU-1390.

\section{References}

Bajaja E., Huchtmeier W.K., Klein U., 1994, A\&A 285, 385 Bresolin F., Capaccioli M., Piotto G., 1993, AJ 105, 1779

Carignan C., Beaulieu S., 1989, ApJ 347, 760

de Vaucouleurs G., 1978, ApJ 224, 710

de Vaucouleurs G., de Vaucouleurs A., Corwin H.G., Buta R.J., Paturel F., Fouque P., 1991, Third Reference Catalogue of
Bright Galaxies. Springer Verlag (RC3)

Fillipenko A., Sargent W.L.W., 1989, ApJ 337, 761

Georgiev Ts.B., Karachentsev I.D., Tikhonov N.A., 1997, Astron. Lett. 23, 586

Karachentsev I.D., Tikhonov N.A., 1994, A\&A 286, 718

Karachentsev I.D., Kopylov A.I., Kopylova F.G., 1994, Bull. Spec. Astrophys. Obs. 38, 15

Landolt A.U., 1992, AJ 104, 340

Makarova L.N., Karachentsev I.D., Georgiev Ts.B., 1997, Astron. Lett. 23, 435

Makarova L.N., Karachentsev I.D., Takalo L.O., Heinämäki P., Valtonen M., 1998, A\&AS (in press)

Sandage A.R., Tammann G.A., 1974, ApJ 191, 559

Sandage A.R., Tammann G.A., 1982, ApJ 256, 339

Sandage A., Bedke J., Atlas of Galaxies Useful for Measuring the Cosmological Distance Scale, 1988, NASA, Washington

Stetson P.B., 1987, PASP 99, 191

Tikhonov N.A., Karachentsev I.D., 1998, A\&A (in press)

van den Bergh S., 1966, AJ 71, 922 